\title{
ESTUDIO CUANTITATIVO DE LA MACROFAUNA DEL SUELO EN DIFERENTES SISTEMAS DE USO DE LA TIERRA EN LA AMAZONÍA PERUANA
}

\section{Beto Pashanasi*}

\section{RESUMEN}

La comunidad de macro-invertebrados del suelo fue evaluada en 22 sistemas de uso del suelo en las zonas de Yurimaguas y Pucallpa. Se separaron, manualmente, 10 muestras por sistema de uso de $25 \mathrm{~cm}$ x $25 \mathrm{~cm}$ x $30 \mathrm{~cm}$ durante la estación lluviosa.

El bosque primario, no intervenido e intervenido, tiene una diversidad muy rica. Asimismo, su densidad (382 a 853 individuos $/ \mathrm{m}^{2}$ ) y su biomasa, dominada por oligochaetas, isópteras y miriápodos $\left(57,8\right.$ a $91,1 \mathrm{~g}$ peso fresco $\left./ \mathrm{m}^{2}\right)$, son bastante altas. Los cultivos de esta comunidad, cuya densidad es de 362 a 574 individuos $/ \mathrm{m}^{2}$ y cuya biomasa es de 5,1 a 32,4 g peso fresco $/ \mathrm{m}^{2}$, se encuentran severamente agotados.

Las pasturas tienen baja diversidad. La densidad de su población varía en un rango de 654 a 1034 individuos $/ \mathrm{m}^{2}$. Su biomasa es tan alta como de 38,4 a 165,9 g peso fresco $/ \mathrm{m}^{2}$, debido a la colonización de la lombriz peregrina, Pontoscolex corethrurus. En las purmas, la densidad poblacional está en un rango de 334 a 838 individuos $/ \mathrm{m}^{2}$; mientras que la biomasa varía entra 4,2 y $102 \mathrm{~g}$ peso fresco $/ \mathrm{m}^{2}$. Cabe destacar que, en algunos casos, la riqueza taxonómica es mayor que la del bosque primario.

Finalmente, los sistemas agroforestales con cobertura de leguminosas tienen la más alta diversidad. Lo contrario ocurre en los sistemas con cobertura de malezas que están por debajo del bosque secundario. Su densidad poblacional se encuentra en un rango que va desde 557 hasta 2896 individuos $/ \mathrm{m}^{2}$, mientras que su biomasa varía entre 18,5 y $170,5 \mathrm{~g}$ peso fresco $/ \mathrm{m}^{2}$, debido a la conservación de gran parte de la fauna del bosque primario que, además, es colonizada por especies oportunistas de terrenos disturbados (miriápodos, oligochaetas e isópteras).

Palabras clave: Macrofauna, suelo, sistemas, bosques, purmas.

* Universidad Nacional de la Amazonía Peruana. Facultad de Zootecnia.Yurimaguas, Loreto (Perú). Correo electrónico: bpashanasi@terra.com.pe 


\begin{abstract}
The community of soils macroinvertebrates was evaluated in 22 different systems of use of the soil, in the zone of Yurimaguas and Pucallpa; by manual separation of 10 samples by system of use of $25 \mathrm{~cm}$ x $25 \mathrm{~cm} \times 30 \mathrm{~cm}$, during the rainy season.

The primary forest not intervened and intervened has great diversity, and a density (382 to 853 individual $/ \mathrm{m}^{2}$ ) and a high biomass $\left(57,8\right.$ to $\left.91,1 \mathrm{~g} / \mathrm{m}^{2}\right)$ dominated by worms, isopteras and myriapods. In cultivations this community is severely exhausted, with a density from 362 to 574 individual $/ \mathrm{m}^{2}$ and a biomass from 5,1 to $32,4 \mathrm{~g} / \mathrm{m}^{2}$.

The pastures have lowers diversity, with density of population in a rank from 654 to 1034 individual $/ \mathrm{m}^{2}$ and a biomass as high as from 38,4 to $165,9 \mathrm{~g} / \mathrm{m}^{2}$, due to the colonization of the «Peregrine worm» Pontoscolex corethrurus. In the fallows purmas the population density is in a rank from 334 to 838 individual $/ \mathrm{m}^{2}$; and a biomass from 4,2 to $102 \mathrm{~g} / \mathrm{m}^{2}$; being the wealth taxonomy greater that of the primary forest in some cases.

Finally the agroforestry systems, with cover of leguminous have the highest diversity, being the contrary thing in the systems with cover of weeds that are under the secondary forest. The population density is found in a rank from 557 to 2896 individual $/ \mathrm{m}^{2}$ and a biomass from 18,5 to $170,5 \mathrm{~g} / \mathrm{m}^{2}$, due to the conservation of great part of the fauna of the primary forest and besides they are colonized for opportunist's species of disturbed lands (myriapodos, worms and isopteras).
\end{abstract}

Key words: Macrofauna, soil, systems, forests, purmas.

\title{
1. INTRODUCCIÓN
}

En los ambientes naturales del trópico húmedo, los macro-invertebrados del suelo son los mejores agentes reguladores de los procesos físico-químicos que afectan la fertilidad de los suelos (Lavelle, 1984; Lee, 1985). Ellos, por acción de la ingestión y deyección del suelo, contribuyen a la conformación de estructuras macro-agregadas resistentes. Los macro-invertebrados mezclan los residuos orgánicos, producto de la ingestión y la deyección, al excavar madrigueras para transportar suelo a la superficie por medio de cámaras subterráneas. 
En los bosques, la diversidad y la abundancia de las comunidades de macro-invertebrados puede ser usada como indicadora de la calidad del suelo (Stork y Eggleton, 1992), toda vez que las lombrices de tierra y la fauna del suelo, en general, influyen en la dinámica de sus procesos químicos. Sin embargo, la naturaleza y los mecanismos de las interacciones entre los microorganismos del suelo y la dinámica de los procesos químicos en los suelos de la Amazonía son aún poco conocidos y, posiblemente, dependientes de las cantidades y calidades de la hojarasca depositada sobre el suelo (Volhland y Schroth, 1999). Según Swift et al., (1979), la tasa de descomposición de los residuos vegetales está influenciada por la calidad del recurso, por los organismos descompositores presentes y por las condiciones ambientales.

En la Amazonía central de Brasil, los «oribatideos» (Oliveira y Franklin, 1993) y los «colémbolos» (Oliveira, 1983) tienen la mayor densidad de la fauna en la hojarasca y en el suelo de áreas no inundables. Las «isópteras» son consideradas importantes descompositoras de la hojarasca (Luizão, 1995) y, en forma conjunta con los «formicides», constituyen los grupos de mayor densidad de la fauna del suelo en la Amazonía central de Brasil (Fittkau y Klinge, 1973; Bandeira y Harada, 1991). Las «oligochaetas» tienen una abundante biomasa en la Amazonía Peruana (Lavelle y Pashanasi, 1989) y en la Amazonía central de Brasil (Barros, 1999). En los varillales de la Amazonía central de Brasil, Luizão (1995) encontró que los «diplópodos»e «isópodos» constituyen una alta densidad y una alta biomasa. De otro lado, en los sistemas agroforestales y barbechos, los «isópodos» presentaron las mayores densidades y biomasas en la macrofauna de la hojarasca, seguidos por los «diplópodos» y por las «isópteras» (Tapia-Coral et al., 1999). Estos organismos, habitantes de la hojarasca y de las capas superiores del suelo, son -debido principalmente a sus interacciones con la microflora-, de importancia crucial para las condiciones de crecimiento de las especies cultivadas y el desenvolvimiento y funcionamiento de los agro-ecosistemas, ya que cumplen un papel vital en la descomposición de la hojarasca y en la liberación de los nutrientes (Swift et al., 1979; Lavelle, 1984; Tian et al., 1997, 1998). Así, una abundante y activa fauna de la hojarasca y del suelo puede ayudar a asegurar un reciclaje rápido de los nutrientes de las plantas (Fittkau y Klinge, 1973), lo que es particularmente importante para áreas cultivadas cuyos insumos son bajos y cuyos suelos son infértiles (Volhland y Schroth, 1999). La capa superficial de hojarasca también confiere protección física al suelo contra la erosión y ayuda en el mantenimiento de su humedad (Ross et al., 1992). De esta forma, dicha capa también está contribuyendo al mantenimiento de la actividad de los organismos del suelo. Por otro lado, estudios recientes demuestran la importancia de la biota del suelo en la recuperación de las áreas degradadas (Barros, 1999; Tapia-Coral et al., 1999; Barros et al., 2000; Araujo-Vergara, 2000; Castilho, 2000). 
Tapia-Coral et al., (1999) encontraron que la cantidad y la calidad de la hojarasca tienen poca influencia sobre la densidad de la macrofauna en sistemas agroforestales de la Amazonía central de Brasil. Sin embargo, la calidad y la cantidad de la hojarasca fueron fuertemente relacionadas con la biomasa de la macrofauna. Asimismo, en la Amazonía central de Brasil, Barros (1999) observó que la calidad de la hojarasca tiene mayor injerencia que su cantidad sobre la riqueza de especies de la macrofauna.

La mayoría de las prácticas de manejo del suelo, independientemente de sus efectos sobre el $\mathrm{pH}$ de este, tiene un efecto negativo sobre su macrofauna. Esto se debe a que las comunidades de la macrofauna del suelo son muy sensibles a los cambios de la cobertura del suelo (Lavelle et al., 1992). En la Amazonía Peruana, Lavelle y Pashanasi (1989) observaron que ocurre un cambio muy drástico en la biomasa y diversidad de los macroartrópodos después de la instalación de pastizales y cultivos anuales.

ICRAF (1996), en un estudio efectuado en Yurimaguas (Perú) en sistemas de multiestratos y plantaciones de Bactris gasipaes con cobertura de Centrosema macrocarpum con 10 años de edad, encontró que ambos sistemas conservaron el mayor número de especies de macro-invertebrados del sistema natural (bosque primario). Dichas especies ofrecían también nichos ecológicos para muchos colonizadores exóticos. Consecuentemente, la recuperación de pastizales degradados a través de sistemas agroforestales puede ser una opción viable para la recolonización de la macrofauna del suelo (Barros et al., 2000).

El objetivo principal fue evaluar el efecto de los principales sistemas de uso de la tierra sobre la comunidad de macro-invertebrados.

\section{MATERIALES Y MÉTODOS}

\subsection{Ubicación}

El estudio fue realizado en las localidades de Yurimaguas y Pucallpa en la Amazonía Peruana entre los meses de noviembre de 1996 y marzo de 1997, durante la estación lluviosa.

La localidad de Yurimaguas está situada en la provincia de Alto Amazonas, departamento de Loreto. Se ubica a $5^{\circ} 56^{\prime}$ latitud sur, $76^{\circ} 5^{\prime}$ longitud oeste y su elevación es de $184 \mathrm{msnm}$. El clima es húmedo tropical, con una temperatura promedio de $26^{\circ} \mathrm{C}$ y una precipitación anual de $2200 \mathrm{~mm}$. Por lo que respecta al clima, esta localidad presenta una estación seca entre los meses de julio y setiembre. Los suelos son 
ultisoles. Su pH es de 4,2 a 4,9; su contenido de arcilla varía en un rango de $4 \%$ a $20 \%$; el contenido de materia orgánica oscila entre $1,7 \%$ a $3 \%$ y su índice de saturación de aluminio va desde $19 \%$ hasta $90 \%$.

La ciudad de Pucallpa se encuentra ubicada a $8^{\circ} 23^{\prime}$ latitud sur, $74^{\circ} 50^{\prime}$ longitud oeste y se eleva $154 \mathrm{msnm}$. El clima es muy cálido, moderadamente lluvioso y con amplitud térmica moderada. La media anual de temperatura es $27^{\circ} \mathrm{C}$, mientras que la precipitación media es de 1500 a $2000 \mathrm{~mm}$. Los suelos son inceptisoles y tienen un un $\mathrm{pH}$ que va desde 4,5 hasta 4,7, así como también un alto índice de saturación de aluminio y un bajo contenido de materia orgánica.

\subsection{Sistemas de uso del terreno (SUT)}

Para el estudio, fueron considerados 11 sistemas de uso de la tierra para cada zona:

- $\quad$ Yurimaguas (Cuadro 1): bosque primario (1); bosque secundario (5); cultivos anuales (1); pastizales (2) y sistemas agroforestales (2).

- $\quad$ Pucallpa (Cuadro 5): bosque primario (2); bosque secundario (2); cultivos anuales y bianuales (4); pastizales (1) y sistemas agroforestales (2).

\subsection{Metodología}

Se obtuvo 10 muestras de cada sistema de uso del suelo. Cada una de ellas, estuvo separada por un intervalo de $5 \mathrm{~m}$ a lo largo de una línea cuyo origen y dirección fueron escogidos al azar. El método de muestreo utilizado fue el recomendado por el Programa Tropical Soil Biology and Fertility (TSBF), (Lavelle, 1984; Anderson e Ingram, 1993), (Figura 1). Cada monolito tuvo las siguientes dimensiones: $25 \mathrm{~cm} \mathrm{x}$ $25 \mathrm{~cm} \times 30 \mathrm{~cm}$. Los monolitos fueron divididos en cuatro estratos sucesivos (hojarasca $0-10 \mathrm{~cm} ; 10-20 \mathrm{~cm} ; 20-30 \mathrm{~cm}$ ) y los invertebrados recolectados fueron conservados en formol al 4\%. En el laboratorio, fueron separados en categorías, órdenes y familias. La densidad fue medida en individuos $/ \mathrm{m}^{2}$ y la biomasa en gramos de peso fresco $/ \mathrm{m}^{2}$. 
Figura 1. Metodología de muestreo por el Tropical Soil Biology and Fertility (TSBF, IUBS/UNESCO) Programme.

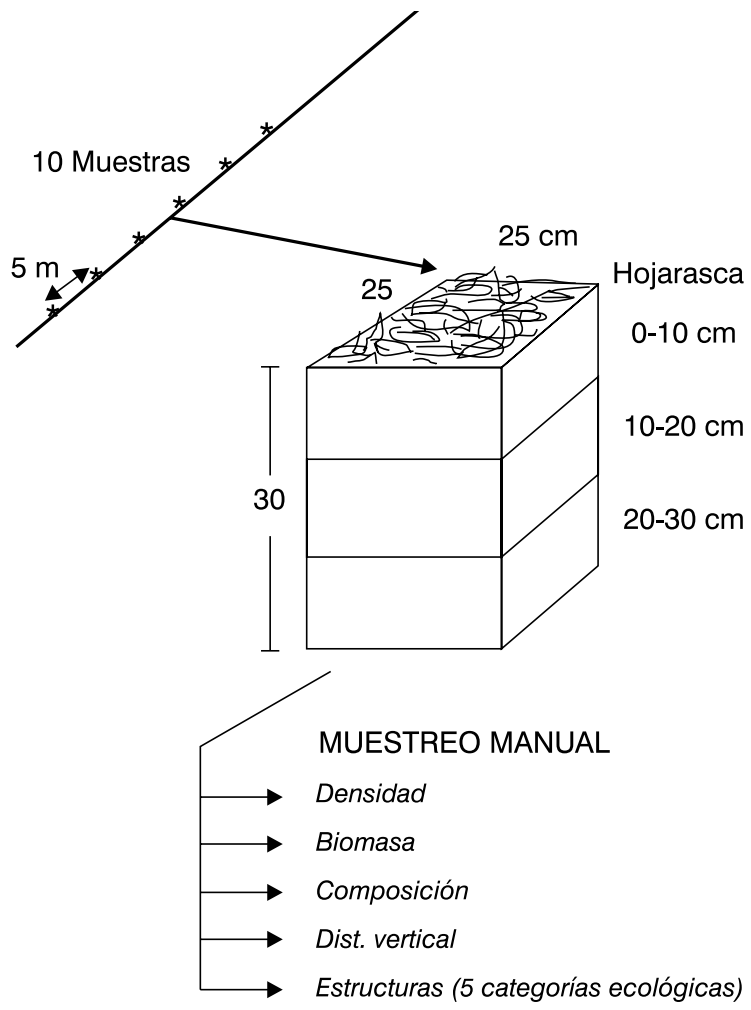

\section{RESULTADOS}

\subsection{Macrofauna del suelo en Yurimaguas}

El bosque primario tuvo una riqueza taxonómica de 25 unidades y una densidad de 446 individuos $/ \mathrm{m}^{2}$, con una biomasa de $57,9 \mathrm{~g}$ de peso fresco $/ \mathrm{m}^{2}$ (Cuadros 1 y 2). El $43 \%$ de la fauna se encontró en la hojarasca, pero el suelo estuvo densamente colonizado hasta los $20 \mathrm{~cm}$ de profundidad (97,1\%), (Figura 2). La comunidad de oligochaetas estuvo dominada por especies epígeas y anécicas que se alimentan de la hojarasca. Estas, seguidas de los coleópteros $(1,1 \%)$ y las isópteras $(0,95 \%)$, son las mayores componentes de la biomasa $(89,1 \%)$. 
El bosque secundario de 20 años tuvo una riqueza taxonómica de 30 unidades y una población de individuos casi dos veces mayor que la del bosque primario. La distribución vertical estuvo concentrada en la hojarasca y en el estrato de $0-10 \mathrm{~cm}(70,7 \%)$, (Figura 2). Las isópteras representaron el 67,2\% de la población; las formicidas, el $11,5 \%$ y las oligochaetas, el 3,5\%. Pero las oligochaetas presentaron la mayor cantidad de biomasa $(51,4 \%)$, mientras que los miriápodos solo el $6,6 \%$.

Figura 2. Distribución en profundidad de los individuos en los diferentes sistemas de uso del terreno en Yurimaguas-Perú.
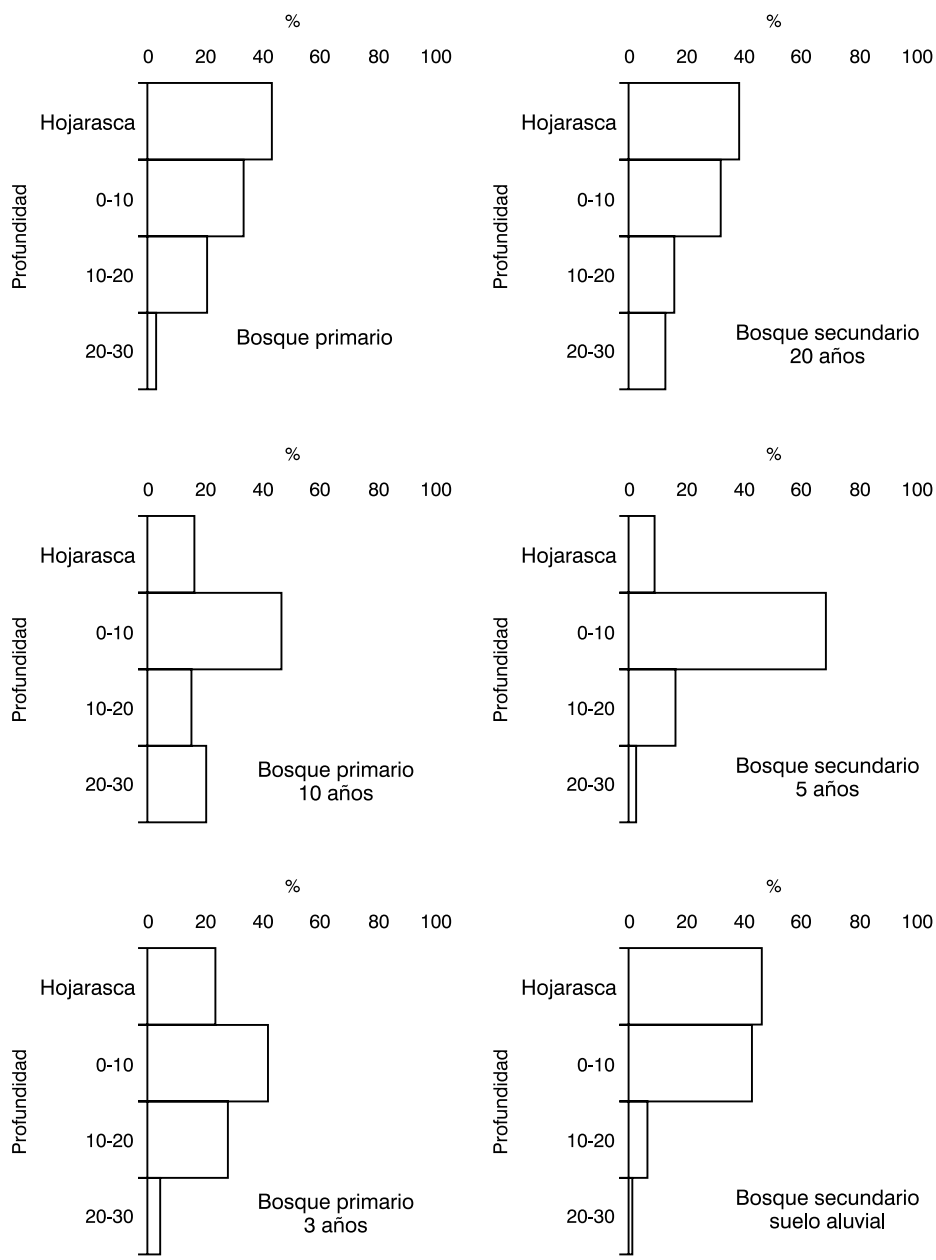
La riqueza taxonómica del bosque secundario de 10 años fue de 20 unidades taxonómicas, con una densidad poblacional de 703 individuos $/ \mathrm{m}^{2}$ y una biomasa de $33,9 \mathrm{~g}$ peso fresco $/ \mathrm{m}^{2}$. La mayor concentración de individuos estuvo localizada en la capa de $0-10 \mathrm{~cm}(46,0 \%)$, (Figura 2). El 48,6\% del total de la población estuvo formado por formicidas; el $22,2 \%$, por isópteras y el $14,5 \%$, por oligochaetas (Cuadros 1 y 2). La mayor cantidad de biomasa está conformada por oligochaetas $(75,2 \%)$. El $84,6 \%$ de ellas corresponde a la Pontoscolex corethrurus, lombriz típica de suelos disturbados.

Por lo que respecta a los bosques secundarios de 3 y 5 años, la riqueza taxonómica, similar a la del bosque secundario de 20 años, fue de 29 unidades. Asimismo, la densidad fue de 485 y 838 individuos $/ \mathrm{m}^{2}$ y la biomasa de 72,7 y $102,0 \mathrm{~g}$ de peso fresco $/ \mathrm{m}^{2}$, respectivamente. En el bosque secundario de cinco años, el $70 \%$ de la población se encuentra en la capa de $0-10 \mathrm{~cm}$, mientras que, en el bosque secundario de tres años, el 40\% de la población se encuentra en la capa de 0-10 cm (Figura 2). Las isópteras conforman el 29,3\% y 61,6\% del total de la población, respectivamente en ambos bosques. Por su parte, las oligochaetas presentaron la mayor biomasa en ambos bosque secundarios ( $82,2 \%$ y 90,6\%, respectivamente), (Cuadros 1 y 2 ).

El bosque secundario de suelos aluviales presenta, con 18 unidades, la más baja riqueza taxonómica, así como también la más baja densidad poblacional (182 individuos $/ \mathrm{m}^{2}$ ) y una biomasa de $5,9 \mathrm{~g}$ peso fresco $/ \mathrm{m}^{2}$ (Cuadros 1 y 2). En la distribución vertical, la mayor concentración de individuos se encuentra en la hojarasca $(47,4 \%)$ y en el estrato de $0-10 \mathrm{~cm}(43,9 \%)$, (Figura 2). El 37,9\% de la población está formado por formicidas y el $27,4 \%$, por oligochaetas epígeas y polyhumicos, que se caracterizan por estar presentes en los lugares donde hay mayor abundancia de materia orgánica.

La riqueza taxonómica en el sistema de los cultivos, similar a la del bosque primario, fue de 25 unidades; su población, de 397 individuos $/ \mathrm{m}^{2}$ y su biomasa, de 32,4 g de peso fresco $/ \mathrm{m}^{2}$. La mayor concentración en la distribución vertical está ubicada en el estrato de $0-10 \mathrm{~cm}(38,7 \%)$, (Figura 3). Las isópteras, las más notables componentes de la población, representan el 44,6\% de ella, las formicidas, el 29,5\%. El 61,1\% de la biomasa está compuesto de oligochaetas (epígeas, anécicas y Pontoscolex corethrurus).

Los pastizales, natural y mejorado, tienen una riqueza taxonómica de 22 y 23 unidades, respectivamente y una biomasa de 57,2 y $165,9 \mathrm{~g}$ peso fresco $/ \mathrm{m}^{2}$ (Cuadros 1 y 2), también respectivamente. El 47,0 y 72,1\% del total de la población está formado por oligochaetas, de las cuales Pontoscolex corethrurus representa el 27,9 y el 67,7\%, 
respectivamente en cada pastizal. Además, los oligochaetas son los mayores componentes de la biomasa (94,4 y 97,4\%, respectivamente). Otros grupos de importancia en la población de individuos son las isópteras $(31,6 \%)$, formicidas $(15,7 \%)$ en el pastizal natural y las isópteras $(19,4 \%)$ en el pastizal mejorado. La mayor concentración de individuos, para ambos sistemas, se encuentra en el estrato de $0-10 \mathrm{~cm}$, que alberga el 68,3\% y 93,2\% del total de la población, respectivamente (Figura 3).

Cuadro 1. Riqueza taxonómica, promedio de abundancia y biomasa de macro-invertebrados en diferentes tipos de uso del terreno en Yurimaguas (Perú).

\begin{tabular}{|l|c|c|c|c|c|}
\hline Sistema de uso del terreno (SUT) & $\begin{array}{c}\mathrm{N}^{0} \text { de } \\
\text { Unid. } \\
\text { Taxon. }\end{array}$ & $\begin{array}{c}\text { Prom. } \\
\text { poblac. } \\
\text { Densid/m² }\end{array}$ & $\begin{array}{c}\text { S.E. de } \\
\text { Densid. }\end{array}$ & $\begin{array}{c}\text { Prom. } \\
\text { biomasa } \\
\text { g.p.f } / \mathrm{m}^{2}\end{array}$ & $\begin{array}{c}\text { S.E. } \\
\text { biomasa }\end{array}$ \\
\hline BOSQUES & & & & & \\
\hline Bosque primario & 25 & 446 & 7,1 & 57,8 & 893,9 \\
\hline PURMAS & & & & & \\
\hline Bosque secundario (20 años) & 30 & 806 & 10,7 & 42,9 & 606,7 \\
\hline Bosque secundario (10 años) & 20 & 703 & 20,5 & 33,0 & 430,6 \\
\hline Bosque secundario (5 años) & 29 & 838 & 16,7 & 101,9 & 680,7 \\
\hline Bosque secundario (3 años) & 29 & 485 & 6,8 & 72,7 & 818,5 \\
\hline $\begin{array}{l}\text { Bosque secundario de suelos } \\
\text { aluviales frecuentemente inundados }\end{array}$ & 18 & 181 & 2,0 & 5,9 & 45,3 \\
\hline CULTIVOS & & & & & \\
\hline Cultivo anual (maíz) & 25 & 397 & 8,0 & 32,4 & 511,6 \\
\hline PASTURAS & & & & & \\
\hline $\begin{array}{l}\text { Pastizal natural degradado } \\
\text { (30 años), (anualmente quemado) }\end{array}$ & 22 & 657 & 6,2 & 57,3 & 330,0 \\
\hline $\begin{array}{l}\text { Pastizal mejorado con Brachiaria } \\
\text { decumbens (15 años) }\end{array}$ & 23 & 914 & 5,8 & 165,9 & 777,1 \\
\hline SISTEMAS AGROFORESTALES & & & & & \\
\hline Plantación de pijuayo (15 años) & 32 & 900 & 9,1 & 84,9 & 843,4 \\
\hline Sistema de multiestratos Bactris/ & & & & & \\
\hline Cedrelinga/Inga/Collubrina & 31 & 557 & 5,7 & 55,7 & 534,4 \\
\hline
\end{tabular}

S.E.: Error estándar 


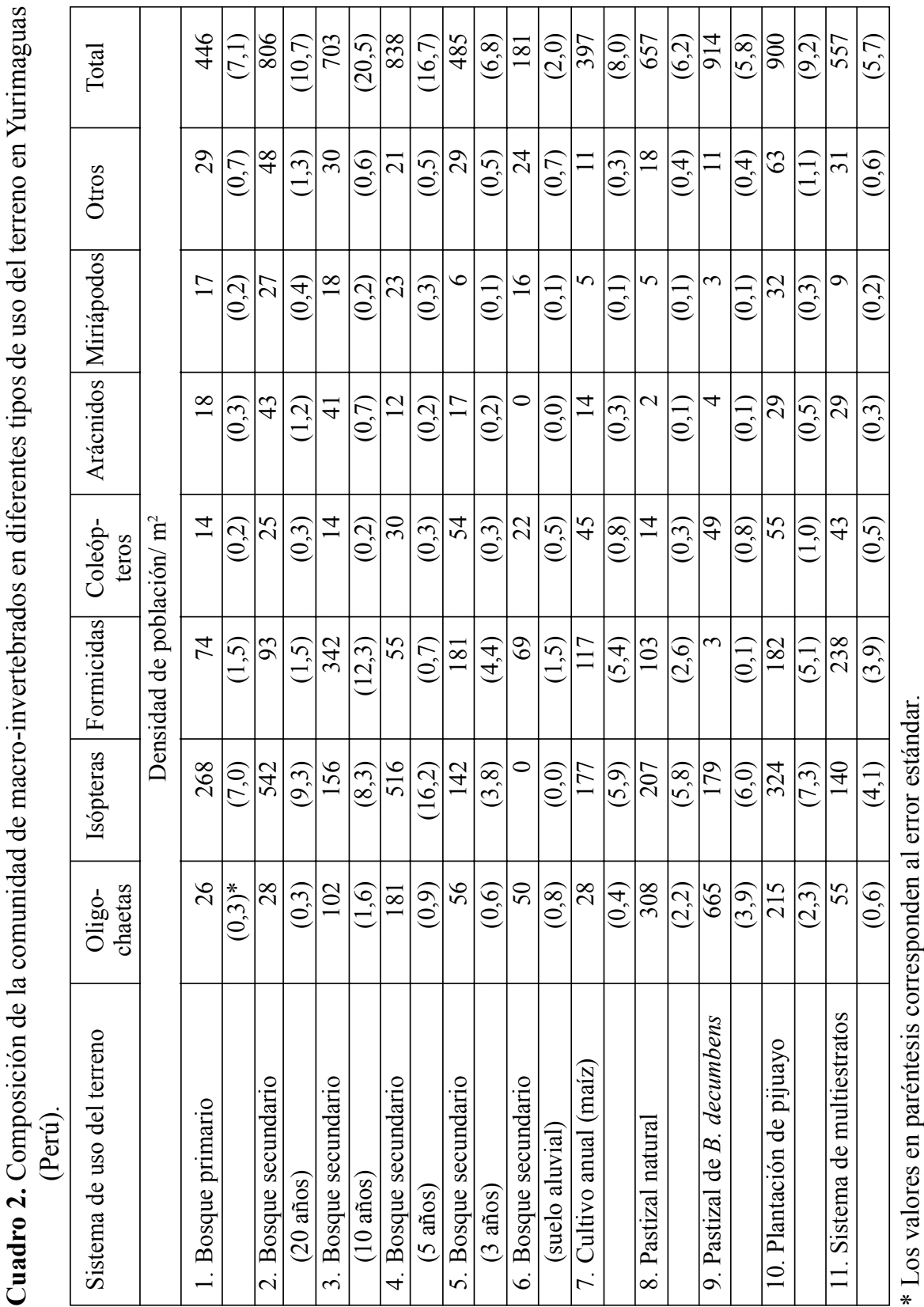




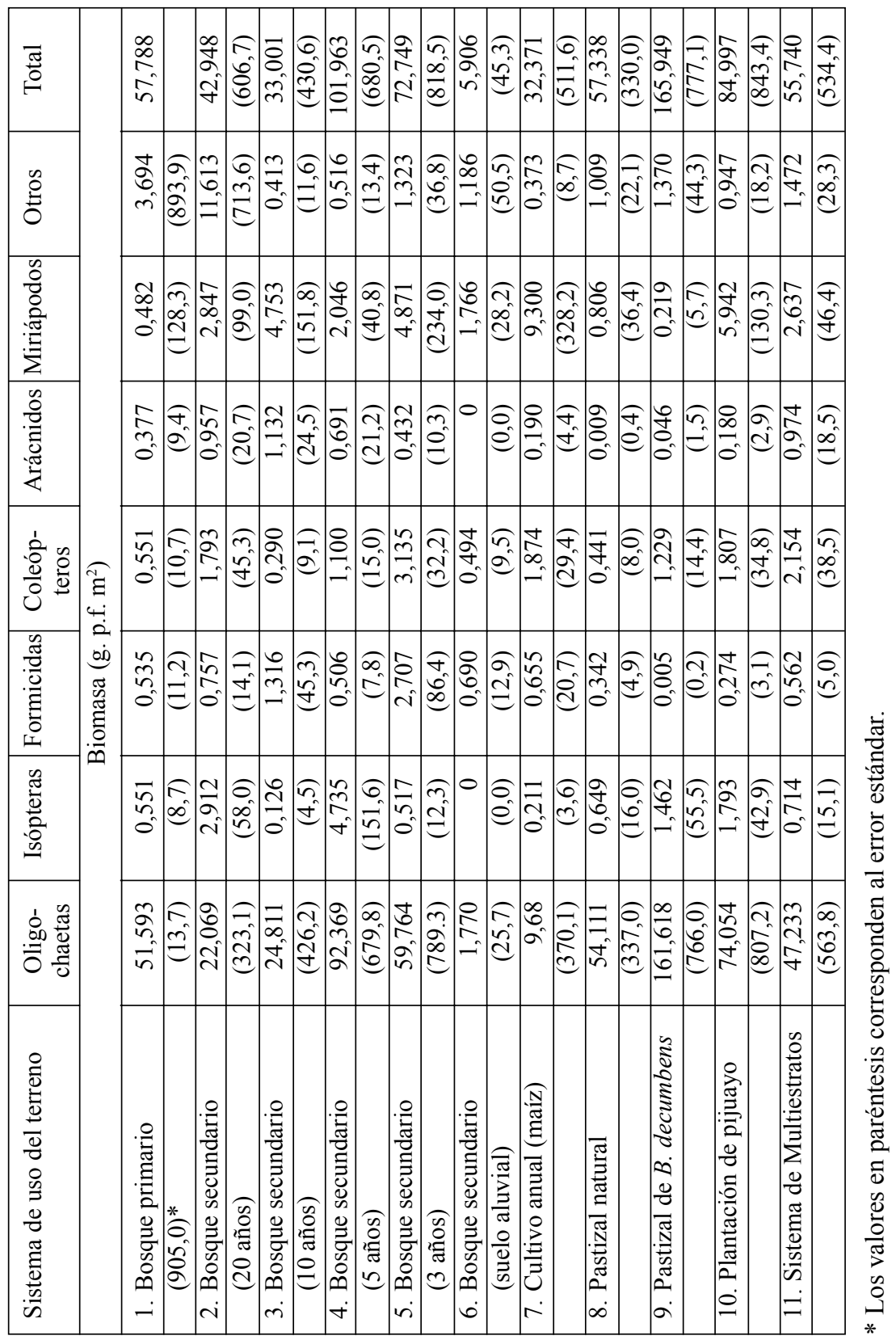


Figura 3. Distribución en profundidad de los individuos en los diferentes sistemas de uso del terreno en Yurimaguas (Perú).
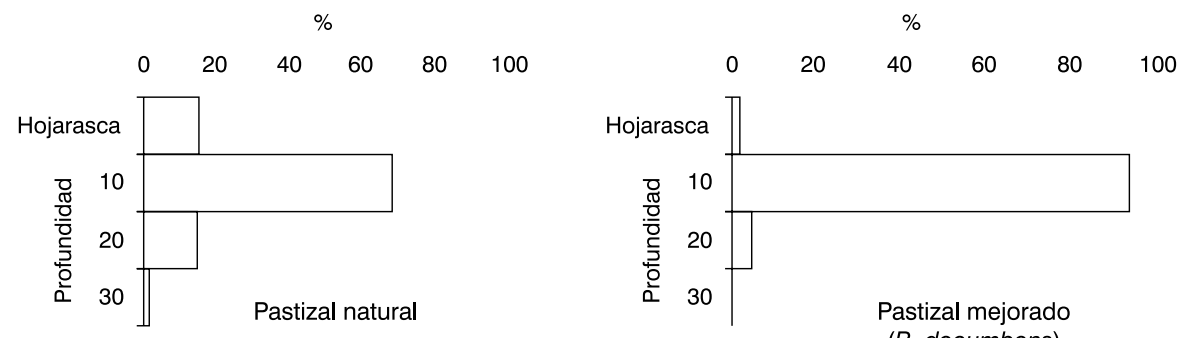

(B. decumbens)
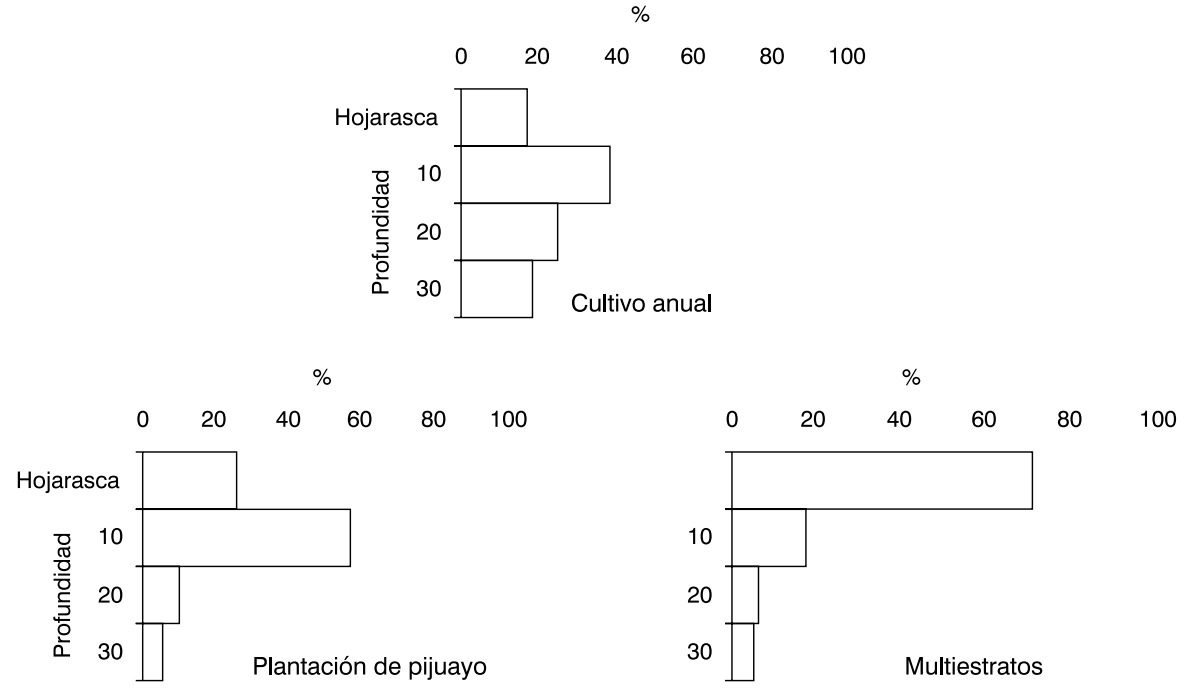

Los sistemas agroforestales están formados por plantaciones de pijuayo y un sistema de multiestratos con asociación de árboles maderables y cultivos perennes, que tienen una riqueza taxonómica de 32 y 31 individuos, respectivamente. La densidad de la población, para ambos sistemas, es de 557 y 901 individuos $/ \mathrm{m}^{2}$, mientras que la biomasa es de 85,3 y 55,9 g peso fresco/ $\mathrm{m}^{2}$ (Cuadros 1 y 2). En la plantación de pijuayo, la población de individuos está formada por isópteras $(36,0 \%)$, oligochaetas $(23,8 \%)$ y formicidas $(20,2 \%)$. En el multiestratos, el componente más representativo son las formicidas $(42,7 \%)$, a las que les siguen las isópteras $(25,1 \%)$ y oligochaetas 
el $(9,9 \%)$. Pero las oligochaetas poseen la mayor biomasa $(87,1 \%)$ en la plantación de Bactris y el $84,7 \%$ en el sistema de multiestratos.

El 70,4\% de la población de individuos en el sistema de multiestratos se encuentra en la hojarasca, mientras que en la plantación de pijuayo el 57,4\% se encuentra en la capa de $0-10 \mathrm{~cm}$ (Figura 3).

\subsection{Macrofauna del suelo en Pucallpa}

Los bosques primarios, no intervenidos e intervenidos, tienen una riqueza taxonómica de 26 y 25 unidades, una densidad poblacional de 382 y 853 individuos $/ \mathrm{m}^{2}$ y una biomasa de 84,9 y $91,1 \mathrm{~g}$ peso fresco $/ \mathrm{m}^{2}$ (Cuadros 3 y 4 ), en todos los casos, respectivamente. El 17,6 \% de la población en el bosque primario no intervenido se encuentra en la hojarasca y en el estrato de $0-20 \mathrm{~cm}(75,3 \%)$. El bosque primario intervenido alberga al $72,2 \%$ del total de la población en la capa de $0-10 \mathrm{~cm}$; solo el $16,9 \%$ se encuentra en la hojarasca (Figura 4). Las formicidas conforman el 66,5\%; las oligochaetas, el 9,7\% y los coleópteros, el 7,1\% del total de la población en el bosque primario no intervenido. Del total de la población en el bosque primario intervenido, los principales componentes son: oligochaetas $(33,2 \%)$, isópteras $(28,1 \%)$ $\mathrm{y}$ formicidas $(23,7 \%)$.

Los bosques secundarios de 3 y 20 años tienen una riqueza taxonómica de 20 y 28 unidades, una densidad de 338 y 523 individuos $/ \mathrm{m}^{2}$ y una biomasa de 4,2 y 105,2 g.p.f. $\mathrm{m}^{2}$ (Cuadros 3 y 4), en todos los casos, respectivamente. En el bosque secundario de 20 años, el 35,2\% de la población corresponde a las isópteras, que están seguidas por las formicidas (31,5\%). Las oligochaetas conforman la mayor cantidad de biomasa $(71,1 \%)$. La población más representativa del bosque secundario de tres años está conformada por las isópteras $(32,2 \%)$, a las que les siguen las oligochaetas $(27,8 \%)$, cuya biomasa $(52,3 \%)$ es la más alta. En la distribución vertical en el bosque secundario de 20 años, el 76,5\% de la población se encuentra en la capa de 0-10 $\mathrm{cm}$; en el bosque secundario de tres años, el $72 \%$ de la población está localizado en la capa de 0-20 cm (Figura 4).

En los cultivos, se observa un decrecimiento dramático en la riqueza taxonómica (15 a 21 unidades); su densidad poblacional es de 362 a 574 individuos $/ \mathrm{m}^{2}$ y su biomasa, de 5,1 a $30,5 \mathrm{~g}$. peso fresco $/ \mathrm{m}^{2}$. En todos los cultivos, las isópteras son el componente más representativo (29,3 a 48,5\%). A estas les siguen las formicidas (10,1 a 22,6\%) y las oligochaetas $(6,1$ a $21,4 \%)$. Las oligochaetas son el componente más notable de la biomasa (4,9 a 93,6\%), (Cuadros 3 y 4). 
En la distribución vertical, la mayor concentración de individuos en los cultivos de maíz y plátano se encuentra en el estrato de $0-20 \mathrm{~cm}(72,1 \%$ y $71,0 \%$, respectivamente), (Figura 4). En los cultivos de arroz y yuca, la mayor concentración de individuos se encuentra en la capa de $0-10 \mathrm{~cm}(39,3 \%$ y 54,9\%, respectivamente), (Figura 4).

En el pastizal mejorado, se observa un decrecimiento de la riqueza taxonómica (18 unidades), una población de 1034 individuos $/ \mathrm{m}^{2}$ y una biomasa de $38,4 \mathrm{~g}$ peso fres$\mathrm{co} / \mathrm{m}^{2}$ (Cuadros 3 y 4). En la distribución vertical, la mayor concentración de individuos está en la capa de 0-10 cm (64,1\%), (Figura 5). La población está dominada por las isópteras $(72,9 \%)$, a las que les siguen las oligochaetas $(13,5 \%)$. La mayor biomasa la conforman las oligochaetas $(83,8 \%)$, seguidas por las isópteras $(5,3 \%)$.

Cuadro 3. Riqueza taxonómica, promedio de abundancia y biomasa de macro-invertebrados en diferentes tipos de uso del terreno en Pucallpa (Perú).

\begin{tabular}{|l|c|r|r|r|r|}
\hline Sistema de uso del terreno (SUT) & $\begin{array}{c}\mathrm{N}^{\mathbf{0}} \text { de } \\
\text { Unid. } \\
\text { Taxon. }\end{array}$ & $\begin{array}{c}\text { Prom. } \\
\text { poblac. } \\
\text { Densid/m² }\end{array}$ & $\begin{array}{r}\text { S.E. de } \\
\text { Densid. }\end{array}$ & $\begin{array}{r}\text { Prom. } \\
\text { biomasa } \\
\text { g.p.f } / \mathrm{m}^{2}\end{array}$ & $\begin{array}{c}\text { S.E. } \\
\text { biomasa }\end{array}$ \\
\hline BOSQUES & & & & & \\
\hline 1. Bosque primario (no intervenido) & 26 & 382 & 10,9 & 84,9 & 1362,7 \\
\hline 2. Bosque primario (intervenido) & 25 & 853 & 10,7 & 91,1 & 692,3 \\
\hline PURMAS & & & & & \\
\hline 3. Bosque secundario (20 años) & 28 & 523 & 12,2 & 105,2 & 1795,7 \\
\hline 4. Bosque secundario (3 años) & 20 & 338 & 5,2 & 4,2 & 85,7 \\
\hline CULTIVOS & & & & & \\
\hline 5. Cultivo anual (arroz) & 19 & 382 & 6,1 & 26,7 & 749,3 \\
\hline 6. Cultivo anual (maíz) & 19 & 574 & 10,7 & 5,1 & 77,4 \\
\hline 7. Cultivo anual (yuca) & 15 & 362 & 7,1 & 30,5 & 814,3 \\
\hline 8. Cultivo bianual (plátano) & 21 & 557 & 12,9 & 8,5 & 182,6 \\
\hline PASTIZAL & & & & & \\
\hline 9. Pastizal mejorado (B. decumbens) & 18 & 1034 & 23,3 & 38,4 & 484,4 \\
\hline SISTEMAS AGROFORESTALES & & & & & \\
\hline 10. Plantación de p. aceitera & 22 & 560 & 9,2 & 18,5 & 287,0 \\
\hline 11. Plantación de caucho & 25 & 2896 & 46,0 & 170,5 & 1300,7 \\
\hline
\end{tabular}

S.E.: Error estándar 
Figura 4. Distribución en profundidad de los individuos en los diferentes sistemas de uso del terreno en Pucallpa (Perú).
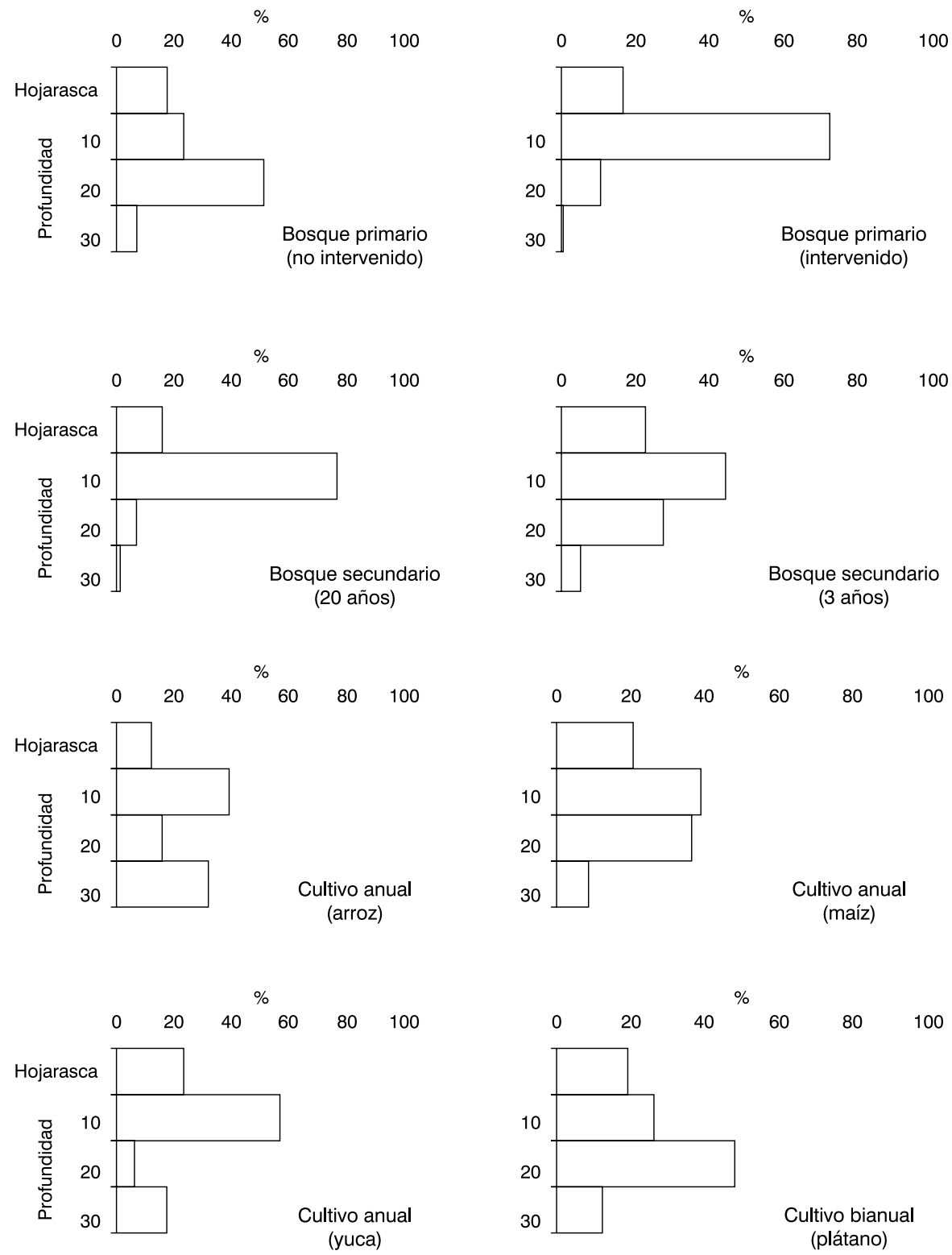


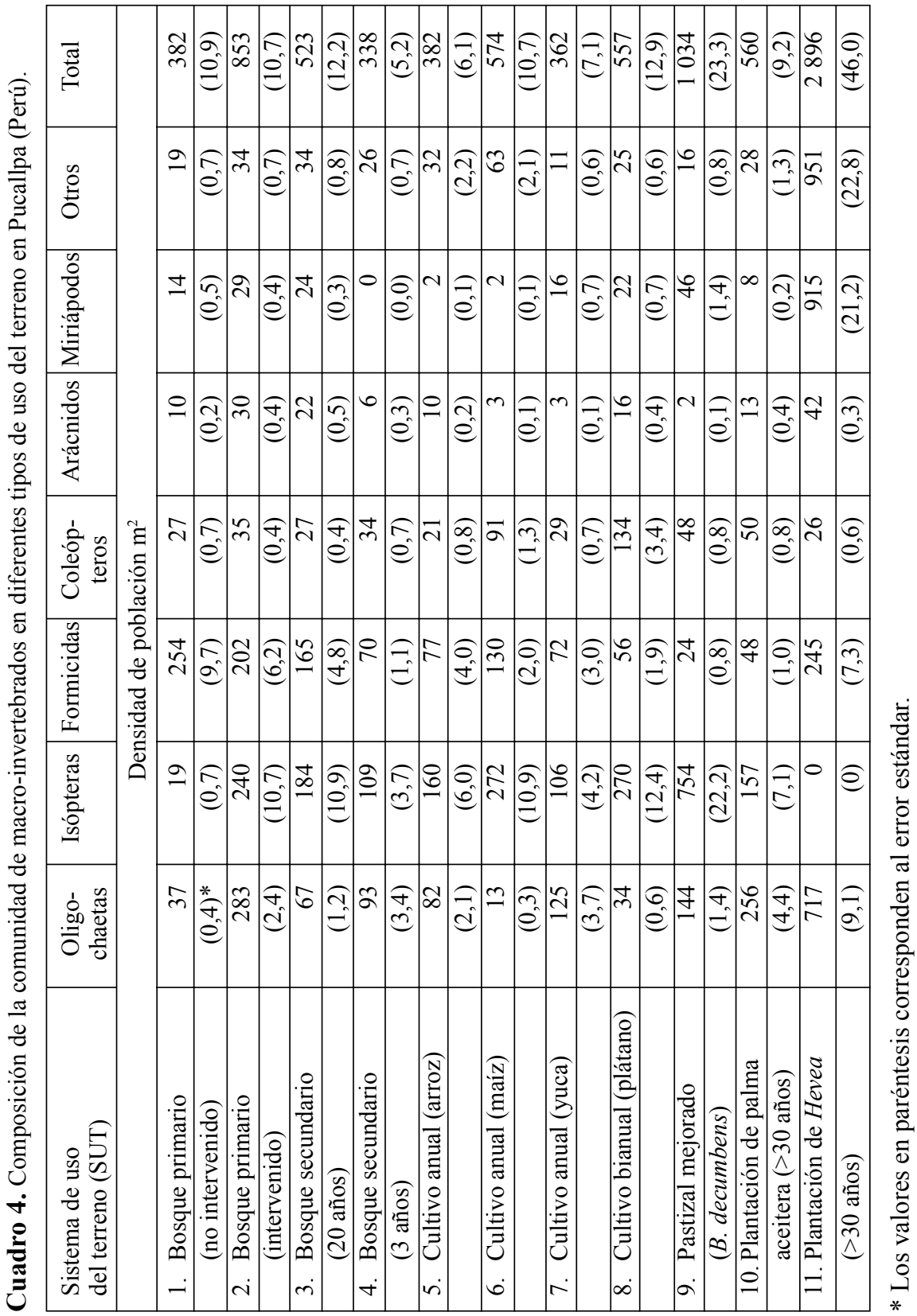




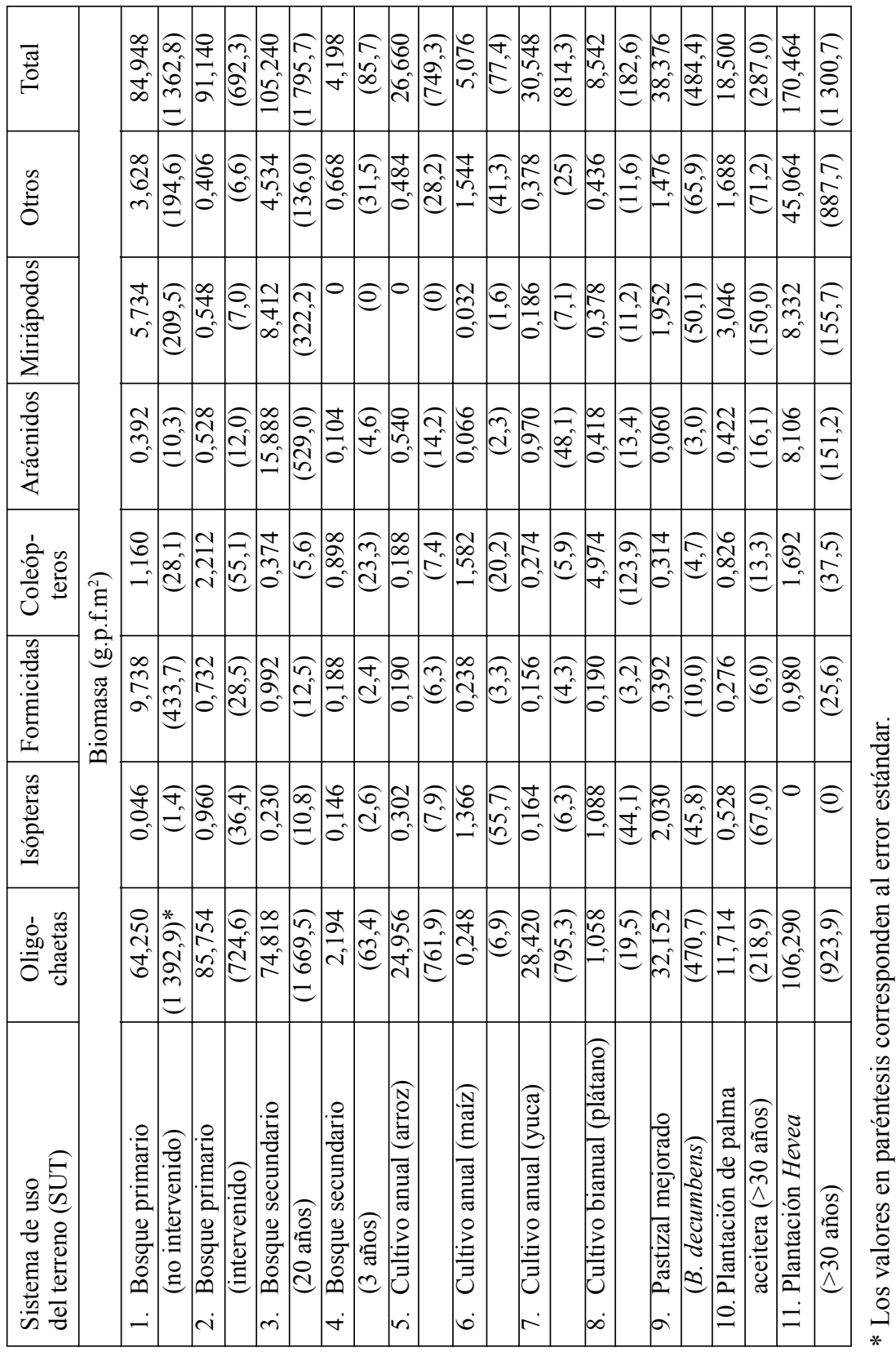


Las plantaciones de palma aceitera y caucho tienen una riqueza taxonómica de 22 y 25 unidades, una densidad poblacional de 560 y 2896 individuos $/ \mathrm{m}^{2}$ y una biomasa de 18,5 y $170,5 \mathrm{~g}$ peso fresco/ $\mathrm{m}^{2}$ (Cuadros 3 y 4), en todos los casos, respectivamente. En la plantación de palma aceitera, el $45,7 \%$ de la población total está formado por oligochaetas y el $28,0 \%$, por isópteras. Los miriápodos $(31,6 \%)$ y las oligochaetas $(24,8 \%)$ son los componentes más representativos en la plantación de caucho. La mayor cantidad de biomasa, en ambas plantaciones, la conforman las oligochaetas 63,3 y $62,4 \%$, en la plantación de palma aceitera y en la de caucho, respectivamente.

Por lo que respecta a la distribución vertical de los individuos, la plantación de palma aceitera es intensamente colonizada hasta $\operatorname{los} 20 \mathrm{~cm}(82,0 \%)$; en cambio, en la plantación de caucho, la hojarasca alberga al $46,4 \%$ de la población y el estrato de $0-10$ $\mathrm{cm}$, al 51,4\% (Figura 5).

Figura 5. Distribución en profundidad de los individuos en los diferentes sistemas de uso del terreno en Pucallpa (Perú).
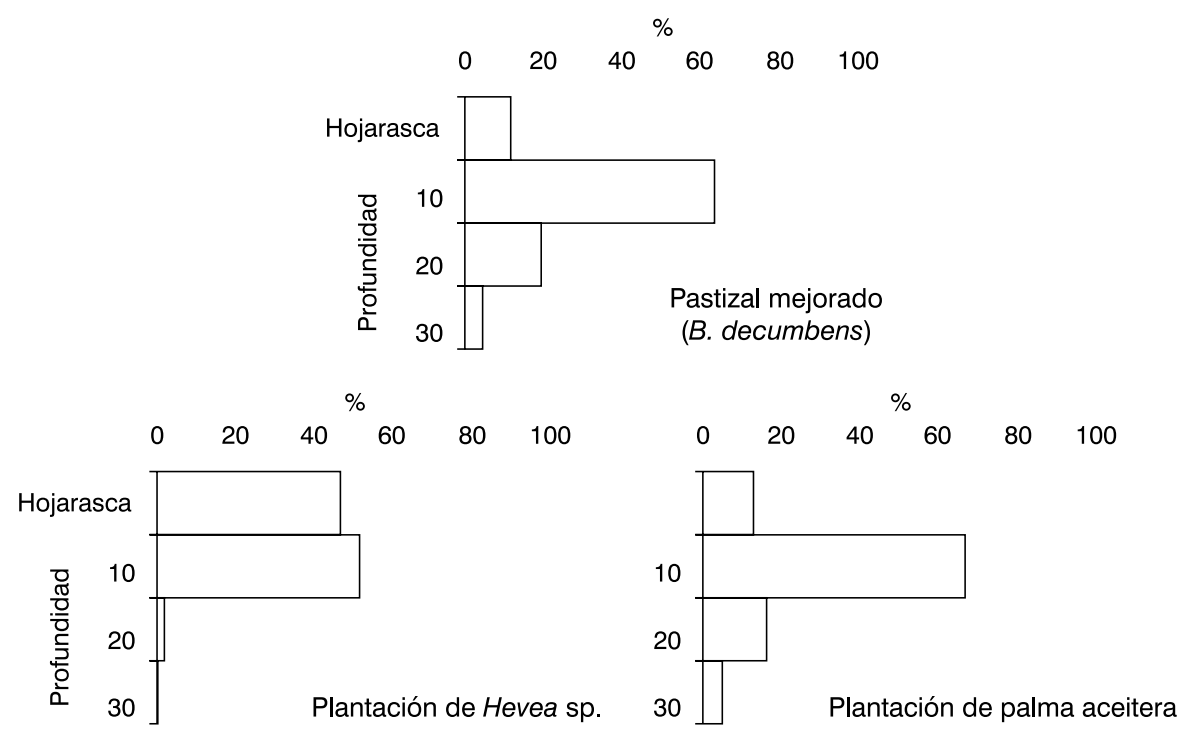


\section{DISCUSIÓN}

Las comunidades de macro-invertebrados de Yurimaguas y Pucallpa fueron afectadas por el sistema de uso de la tierra. Los bosques primarios, no intervenidos e intervenidos, tienen una diversidad muy similar a la de las purmas, una densidad de 382 a 853 individuos $/ \mathrm{m}^{2}$ y una biomasa de 57,8 a $91,9 \mathrm{~g}$ peso fresco $/ \mathrm{m}^{2}$, lo que es muy similar a lo encontrado en el medio ambiente de México (Lavelle et al., 1981; Lavelle y Kohlmann, 1984), de Nigeria (Madge, 1969) y en el sudeste de Asia (Collins, 1980; Leakey y Proctor, 1987). Sin embargo, Tapia-Coral y Pashanasi (2001) encontraron valores de dos a tres veces más altos en el bosque primario de Jenaro Herrera (2 482 individuos $/ \mathrm{m}^{2}$ ). Asimismo, Lavelle y Pashanasi (1989) obtuvieron valores aun más altos para la diversidad y la densidad en condiciones similares.

El desmonte y los cultivos sucesivos destruyen rápidamente al mayor número de invertebrados dentro de la fauna. En áreas cultivadas con cultivos anuales y bianuales, con relación a los bosques primarios, la biomasa fue reducida al 56,1\%, en Yurimaguas, y entre 6,0 a 35,9\%, en Pucallpa. La mitad de las unidades taxonómicas desapareció.

El pastizal mejorado tiene la más alta biomasa en Yurimaguas (169,9 g/peso fresco/ $\left.\mathrm{m}^{2}\right)$, pero una biomasa más baja en Pucallpa $\left(38,4 \mathrm{~g}\right.$ peso fresco $\left./ \mathrm{m}^{2}\right)$. Su riqueza taxonómica es de 18 a 23 unidades, debido al gran desarrollo de poblaciones de Pontoscolex corethrurus, que representa del 97,4 al 83,8 \% de la biomasa total. Estos resultados son similares a los encontrados por Lavelle y Pashanasi (1989).

El pastizal de Pucallpa tiene una baja riqueza taxonómica, debido a que este es quemado anualmente en la época seca.

Las pasturas son favorables para el desarrollo de numerosas poblaciones de lombrices (Pontoscolex corethrurus). Esto confirma anteriores observaciones realizadas en pastizales naturales y mejorados (Lavelle y Pashanasi, 1989), donde se registraron biomasas de 800 a $1600 \mathrm{~kg}$ de peso fresco por hectárea. En las pasturas, que son colonizadas por lombrices exóticas, desaparecen todas las lombrices nativas.

Los bosques secundarios de diferentes edades de Yurimaguas tienen una comunidad de macro-invertebrados menor o similar a la de los bosques primarios. La comunidad menos numerosa se encuentra en el bosque secundario aluvial, debido a su inundación anual. La densidad está en el orden del 485 a 838 individuos $/ \mathrm{m}^{2}$. Esta es dos veces mayor en el bosque secundario de cinco años que en el bosque primario. La mayor riqueza taxonómica se encuentra en el bosque secundario de 20 años y la más baja en el suelo aluvial. En Pucallpa, la densidad y la riqueza taxonómica son simila- 
res. En relación con la biomasa, la dominancia de lombrices es relativamente baja, pero se observa una población constante de Pontoscolex corethrurus, que normalmente está ausente en el bosque primario.

Finalmente, los sistemas agroforestales con plantaciones de Bactris gasipaes y multiestratos con cobertura de Centrosema macrocarpum en Yurimaguas tienen una excelente comunidad de lombrices (Pontoscolex corethrurus) de bosque primario y de pastizales, así como también una alta densidad de termitas y epigeas detritívoras de la hojarasca. Las plantaciones con cobertura de malezas de Hevea sp. y Eleais guianensis, en Pucallpa, tienen una riqueza similar a la del bosque primario (22 a 25 unidades), además de una excelente población de miriápodos, isópteras y oligochaetas.

Estos resultados muestran, claramente, el efecto perjudicial de los cultivos sobre la macrofauna del suelo, sobre todo para las lombrices de todas las categorías ecológicas y fauna epígea de la hojarasca. Las isópteras son las más resistentes y presentan una mayor abundancia.

\section{AGRADECIMIENTO}

Este estudio fue hecho como parte del Programa Tropical Soil Biology and Fertility (TSBF), desarrollado en Yurimaguas (Perú). Nuestro sincero agradecimiento al doctor Mike Swift, jefe del programa, por su apoyo material y económico para la ejecución del presente trabajo.

\section{BIBLIOGRAFÍA}

ANDERSON, J.M.; INGRAM, J.S. 1993. Tropical Soil Biology and Fertility: A Handbook of Methods. CAB International, Wallingford, UK. 256 pp.

ARAUJO-VERGARA, Y.M. 2000. Oligoquetos Sob Adição de Liteira e sua Relação com a Disponibilidade de Nitrogênio em Solos de Capoeira na Amazônia Central. Dissertação de Mestrado. Manaus, AM.: INPA/FUA. 88 pp.

BANDEIRA, A.G.; HARADA, A.Y. 1991. Cupins e Formigas na Amazônia. En: VAL, A.L.; FIGLIUOLO, R.; FELDBERG, E. (ed.). Bases Científicas para Estratégias de Preservação e Desenvolvimento da Amazônia: Fatos e Perspectivas. Vol 1. Manaos: Instituto Nacional de Pesquisas da Amazônia. pp 387-395. 
BARROS, E. 1999. Effet de la Macrofaune Sur la Structure et les Processus Physiques $d u$ Sol de Paturages Degrades D'Amazonie. Thèse de Doctorat de L’Université Paris 6. France. 127 pp.

BARROS, E.; NEVES, A.; FERNANDES, E.C.M.; WANDELLI, E.; LAVELLE, P. 2000. Soil macrofauna community of Amazonian Agroforestry Systems. Agroforestry Systems (no publicado).

CASTILHO, G. 2000. Efeito da qualidade do substrato na biomassa microbiana do solo duma capoeira da Amazônia Central. Dissertação de Mestrado. Manaus, AM.: INPA/FUA. 55 pp.

COLLINS, N. M. 1980. The distribution of soil macrofauna on the West ridge of Gunung (Mount) Mulu, Sarawak. En: Oecologia. 44: 263-275.

FITTKAU, E.J.; KLINGE, H. 1973. On biomass and trophic structure of the Central Amazonian rain forest. En: Biotropica, 5(1):2-14.

ICRAF (International Center for Research in Agroforestry), Annual Report. 1996. Nairobi, Kenya. pp. 39-69.

LAVELLE, P.; MAURY, M. E.; SERRANO. V. 1981. Estudio cuantitativo de la fauna del suelo en la región de Laguna Verde, Veracruz. Época de lluvias. México: Inst. Ecol. Publ. 675 - 105.

LAVELLE, P. 1984. The Soil Systems Humid Tropics. En: Biology International. 9: 2 - 17.

LAVELLE, P.; KOHLMAN, B. 1984. Etude quantitative de la macrofaune du sol dans une foret tropicale humide du Mexique (Bonampak, Chipas). En: Pedobiología 27:377-393.

LAVELLE, P.; PASHANASI, B. 1989. Soil macrofauna and land management in Peruvian Amazonia (Yurimaguas, Loreto). En: Pedobiologia 33:283-291.

LAVELLE, P.; SPAIN, A. V.; BLANCHART, E.; MARTIN, A.; MARTIN, S. 1992. The impact of soil fauna on the properties of soils in the humid tropics. En: Myths and Science of Soils of the Tropics. SSSA Special Publication. Madison Wisconsin. pp. 157-185. 
LEAKEY, R. J.; PROCTOR, G. 1987. Invertebrates in the litter and soil at a range of altitudes on Gunung Silam, a small ultrabasic mountain in Sabah. J. En: Trop. Ecol. 3 119-129.

LEE, K. E. 1985. Earthworms: Their Ecology and Relationships with Soils and Land Use. London: Acad. Press. 411 pp.

LUIZÃO, F. J. 1995. Ecological Studies in Contrasting Forest Types in Central Amazonia. Ph. D. Thesis. Stirling, UK: University of Stirling. 288 pp.

MADGE, D. S. 1969. Field and laboratory studies on the activities of two species of tropical earthworms. En: Pedobiología 9:188-214.

OLIVEIRA, E.P. 1983. Colêmbolos (Insecta: Collembola) epigêicos como indicadores ecológicos de ambientes florestais. Dissertação de Mestrado. Manaus, AM.: INPA/FUA. 105 pp.

OLIVEIRA, E.P.; FRANKLIN, E. 1993. Efeito do fogo sobre a mesofauna do solo. Recolonização de áreas queimadas. En: Pesquisa Agropecuária Brasileira 28 (3):357-369.

ROSS, S.M.; LUIZÃO, F.J.; LUIZÃO, R.C.C. 1992. Soil conditions and soil biology in different habitats across a forest-savanna boundary on Maracá Island, Roraima, Brazil. En: FURLEY, P.A.; PROCTOR, J; RATTER, J.A. (ed.). Nature and Dynamics of Forest-Savanna Boundaries. London: Chapman \& Hall. pp:145-170.

STORK, N.E.; EGGLETON, P. 1992. Invertebrates as determinants and indicators of soil quality. En: Amer. Jour. Altern. Agricul. 7:38-47.

SWIFT, M.J.; HEAL, O.W.; ANDERSON, J.M. 1979. Decomposition in terrestrial Ecosystems. En: Studies in Ecology. $\mathrm{N}^{\circ}$ 5. Berkeley: University of California Press.

TAPIA-CORAL, S.C.; LUIZÃO, F.; WANDELLI, E. 1999. Macrofauna da liteira em sistemas agroflorestais sobre pastagens abandonadas na Amazônia central. En: Acta Amazônic, 29 (3): 477-495. 
TAPIA-CORAL, S.C; PASHANASI, B. 2001. Macrofauna del suelo en los varillales y chamizales de la Amazonía Peruana (No publicado).

TIAN, G.; BRUSSAARD, L.; KANG, B.T.; SWIFT, M.J. 1997. Soil Fauna-mediated decomposition of plant residues under constrained enviromental and residue quality conditions. En: CADISH, G.; GILLER, K.E. (ed.). Driven by Nature: Plant Litter Quality and Decomposition. CAB International. UK. pp. 125134.

TIAN, G.; ADEJUYIGBE, C.O.; ADEOYE, G.O.; KANG, B.T. 1998. Role of soil microarthropods in leaf decomposition and $\mathrm{N}$ release under various landuse practices in the humid tropics. En: Pedobiologia 42:33-42.

VOLHLAND, K.; SCHROTH, G. 1999. Distribution patterns of the litter macrofauna in agroforestry and monoculture plantations in Central Amazonia as affected by plant species and management. En: Applied Soil Ecology, (13): 57-68. 